\title{
Effects of Densities of Brominated Flame Retardants on the Detection Response for HPLC Analysis with a Corona-charged Aerosol Detector
}

\author{
Shigetomo Matsuyama, ${ }^{*}, * *$ Yukari Orihara, ${ }^{*}$ Shinichi Kindgasa, ${ }^{*}$ and Hajime Ohtani** \\ *National Metrology Institute of Japan/National Institute of Advanced Industrial Science and Technology, \\ Higashi 1-1-1, Tsukuba, Ibaraki 305-8565, Japan \\ **Nagoya Institute of Technology, Gokiso-cho, Showa, Nagoya 466-8555, Japan
}

\begin{abstract}
Dependence of the response of a corona-charged aerosol detector (corona CAD) on the concentration and densities of brominated flame retardants and some related substrates was studied. The calibration curves of the substrates did not show linearity and the substrate with a lower density exhibited the stronger response. Regardless of the solvents (chloroform or toluene), and the injected volume of the substrate solution, the signal intensity of the substrate observed by a corona CAD was substantially proportional to $2 / 3$ power law of concentration and proportional to $(-2 / 3)$ power law of the density of the substrates. These results suggest that the responses should be proportional to the surface area of the particles generated through the drying process in corona CAD. Contrary to the former reports that the detector response of a corona CAD was independent of chemical species, it was proved that the response varies with the density of a substrate.
\end{abstract}

Keywords Corona-charged aerosol detector, HPLC, density, concentration, response, brominated flame retardants

(Received October 25, 2014; Accepted December 9, 2014; Published February 10, 2015)

\section{Introduction}

High performance liquid chromatography (HPLC) is nowadays one of the most widespread methods used in analytical laboratories. ${ }^{1}$ A UV/vis detector is the most common apparatus for HPLC. However, the analytes without suitable chromophor or fluorophor are not appliciable for UV/vis and fluorescence detection. $^{2}$ Although a refractive index detector responds for any analyte, its sensitivity is not sufficient and it cannot be used for the gradient technique. Mass spectrometer, which can also nonspecifically detect various analytes, needs careful maintenance to keep the system clean. As such, an alternative nonspecific detector to overcome these issues has been desired.

Recently, several evaporation/aerosol based detectors, such as evaporative light scattering detector (ELSD), ${ }^{3-4}$ nano quantity analyte detector (NQAD) ${ }^{5}$ and corona-charged aerosol detector (corona CAD) ${ }^{6-8}$ were developed. These apparatus can detect the nonvolatile species independent of their chemical structures and are claimed to provide a universal response for the

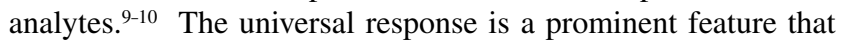
enables us to make quantitative analyses without having to prepare individual calibrations even for newly synthesized or unknown compounds. ${ }^{1,10}$ From the comparison of these detectors, ${ }^{9,11-13}$ the reproducibility of the response from corona CAD is better than two other detectors. ${ }^{14}$ The sensitivity of corona CAD is comparable to that of NQAD and better than

$\dagger$ To whom correspondence should be addressed.

E-mail: s.matsuyama@aist.go.jp that of ELSD. ${ }^{14-16}$ In addition, the response of corona CAD also more universal compared with ELSD. ${ }^{17-18}$ So far, experimental results by Górecki et al. showed that the response factors obtained from nonvolatile species were very close to each other when the composition of the mobile phase was matched. ${ }^{19}$ However, the reason for the universal response of corona CAD for nonvolatile species has not been elucidated theoretically. On the other hand, we reported recently that semi-volatile substrates (a series of phthalates) did not show the universal response, in which the phthalates with higher vapor pressure provided lower sensitivity for corona CAD. ${ }^{20}$ It is important to clarify the conditions under which the universal response of corona CAD can be achieved.

In corona $\mathrm{CAD}$, the mobile phase is nebulized by nitrogen gas to make aerosols, which are introduced into the drying tube at ambient temperature to evaporate the solvent. After the drying process, the particles of a substrate are generated and mixed with ionized nitrogen gas to acquire a charge. The unreacted ionized gas is removed by an ion trap, and then the charged particles generate an electric signal amplifiable by an electrometer. ${ }^{21}$ It is assumed that the response of corona CAD should change depending on the total surface area of the particles because the electrical charge of the particle generally locates on the surface. Even if the mass of the particles are equivalent, their surface area generally varies with the density of the substrate. It is expected, therefore, that the density should affect the response of corona CAD contrary to the generally accepted "universal" response. In the experiments by Górecki et al. ${ }^{19}$ the densities of the examined substrates (sulfamethoxazole, sulfamerazin, sulfamethizol, sulfadmethazine 
Table 1 Substrates analyzed by corona CAD

\begin{tabular}{|c|c|c|c|c|c|}
\hline Code & Substrate & CAS No. & Supplier & Density/g cm $\mathrm{cm}^{-3}$ & Ref. \\
\hline S-01 & Bisphenol A & $80-05-7$ & Sigma-Aldrich Co., LLC. & 1.21 & 22 \\
\hline $\mathrm{S}-02$ & Chlorinated paraffin $70 \%$ & $61788-76-9$ & Wako Pure Chemical Industries, Ltd. & 1.64 & $*$ \\
\hline S-03 & Dechlorane plus & $13560-89-9$ & Occidental Petroleum Corp. & 1.75 & $*$ \\
\hline S-04 & FG-3200 & $25327-89-3$ & Teijin Chemical Ltd. & 1.83 & $*$ \\
\hline S-05 & FG-7500 & $28774-93-8$ & Teijin Chemical Ltd. & 1.85 & $*$ \\
\hline S-06 & Pratherm EP-20 & $68928-70-1$ & DIC Corp. & 1.92 & $*$ \\
\hline S-07 & TBBPA carbonate oligomer BC52 & $94334-64-2$ & Chemtura Corp. & 1.95 & $*$ \\
\hline S-08 & Pratherm EC-20 & - & DIC Corp. & 2.07 & $*$ \\
\hline S-09 & FG-8500 & $71342-77-3$ & Teijin Chemical Ltd. & 2.15 & $*$ \\
\hline S-10 & Tetrabromobisphenol A & $79-94-7$ & Tokyo Chemical Industry Co., Ltd. & 2.16 & 22 \\
\hline S-11 & Hexabromocyclododecane & $3194-55-6$ & Wako Pure Chemical Industries, Ltd. & 2.36 & $*$ \\
\hline S-12 & Pyroguard SR-245 & $25713-60-4$ & DKS Co., Ltd. & 2.54 & $*$ \\
\hline S-13 & Octabromodiphenyl ether & $32536-52-0$ & Great Lakes Chemical Corp. & 2.85 & $*$ \\
\hline S-14 & Pentabromotoluene & $87-83-2$ & Tokyo Chemical Industry Co., Ltd. & 3.19 & $*$ \\
\hline S-15 & Decabromodiphenyl ether & $1163-19-5$ & Sigma-Aldrich Co., LLC. & 3.35 & $*$ \\
\hline S-16 & Hexabromobenzene & $87-82-1$ & Tokyo Chemical Industry Co., Ltd. & 3.54 & 22 \\
\hline P-01 & Polystyrene & $9003-53-6$ & Showa Denko K.K. & 1.05 & 23 \\
\hline P-02 & Poly(methyl methacrylate) & 9011-14-7 & Showa Denko K.K. & 1.19 & 24 \\
\hline
\end{tabular}

Numbers in the reference column $(22,23$ and 24$)$ correspond to numbers in the reference literature. *. Density analyzed by pycnometer.

and sulfaguanidyne) were in a limited range $\left(1.32-1.57 \mathrm{~g} \mathrm{~cm}^{-3}\right) .22$ However, the densities of a variety of substrates are distributed in a much wider range. Especially, the densities of some brominated flame retardants are larger than $3.5 \mathrm{~g} \mathrm{~cm}^{-3}$. In this work, various brominated flame retardants and related substrates were measured with the corona CAD. The observed results were discussed in terms of the effect of the density of the substrates on the response of the corona CAD.

\section{Experimental}

\section{Sample substrates}

The substrates investigated in this study are listed in Table 1 together with their densities and suppliers. The S- code substrates were brominated flame retardants and their related substrates, all of which were non-volatile at ambient temperature. The $\mathrm{P}$ - code substrates were polymers, of which weight average molar mass $\left(M_{\mathrm{w}}\right)$ and the polydispersity $\left(M_{\mathrm{w}} / M_{\mathrm{n}} ; M_{\mathrm{n}}\right.$ is the number average molar mass) were $M_{\mathrm{w}}=28500, M_{\mathrm{w}} / M_{\mathrm{n}}=1.03$ for P-01, and $M_{\mathrm{w}}=20600, M_{\mathrm{w}} / M_{\mathrm{n}}=1.06$ for P-02, respectively.

The densities of the sample substrates without reference values were measured using an ultra pycnometer 1000 (Quantachrome Instruments). A micro sell was used for determining the densities of S-02, S-11, S-13 and S-15, and the sample mass ranged from 1.8 to $4.3 \mathrm{~g}$. The substrates S-03, S-05, S-06, S-07, S-08 and S-09 were analyzed in a meso sell, and the mass ranged from 0.4 to $1.0 \mathrm{~g}$. A nano sell was used for S-04, S-12 and S-14, with mass ranging from 0.15 to $0.26 \mathrm{~g}$. The purging time of helium gas before analysis was $5 \mathrm{~min}$, the gas pressure was $121 \mathrm{kPa}$ and the temperature was about $26^{\circ} \mathrm{C}$. The purity of helium gas used for this experiment was higher than $99.999 \%$. The observed densities are listed in Table 1 along with reference values. ${ }^{22-24}$

\section{HPLC-corona CAD analysis}

All substrates were weighed $(4-27 \mathrm{mg})$ and individually dissolved in chloroform (Wako Pure Chemical Industries, Ltd., HPLC-grade). The obtained solutions were diluted in measuring flasks $\left(5,10\right.$ or $\left.20 \mathrm{~cm}^{3}\right)$ to prepare the mother solutions. Other mother solutions dissolved in toluene for the substrates S-03,
04 , and $10-16$ were also prepared. The concentration range of the mother solutions was $0.4-4.9 \mathrm{mg} \mathrm{cm}^{-3}$. For this operation, vessels with amber glass were used for the photosensitive substrates including $\mathrm{Cl}$ or $\mathrm{Br}$. The mother solutions were further diluted $\left(10-300 \mu \mathrm{g} \mathrm{cm}^{-3}\right)$ and then subjected to the HPLC-corona CAD measuments. An HPLC system (Agilent Technologies Inc., 1260 infinity) consisted of a degasser, a binary analytical pump, an autosampler capable of injection up to $1500 \mathrm{~mm}^{3}$, a thermostatted column compartment, a diode array detector (DAD) and an Agilent Open Lab CDS ChemStation (revision C.01.03) was used in this work. HPLC-grade of chloroform or toluene (Wako Pure Chemical Industries, Ltd.) was used as mobile phases in HPLC-corona CAD analysis. Three resistance stainless tubes (Tosoh Corp., 6783) were connected in a series instead of the separation column to prevent the adsorption of the substrates on the column packings for maintaining a suitable backpressure. The flow rate of the mobile phase was set to $0.5 \mathrm{~cm}^{3} \mathrm{~min}^{-1}$ and the injected volume of the sample solution was 10 or $1000 \mathrm{~mm}^{3}$. Corona CAD (Thermo Fisher Scientific, Inc., Corona CAD ultra RS) was placed in line after the DAD of the HPLC system. The nebulizer gas in the corona CAD was nitrogen and its pressure was maintained at $241 \mathrm{kPa}$. The purity of nitrogen gas used in the corona CAD measurements was higher than $99.9995 \%$.

\section{Syringe pump-corona CAD analysis}

The concentration of a substrate in the eluted solution from HPLC should vary depending on the elution profile. To study the relationship between the signal intensity by corona CAD and the "real" concentration of a substrate, a syringe pump was used to introduce a constant concentration flow of the substrates into the corona CAD. The mother solutions of the substrates (S-03, 04 and 10-16) were diluted with toluene (Wako Pure Chemical Industries, Ltd., HPLC-grade) in a $10 \mathrm{~cm}^{-3}$ measuring flask to make the sample solutions. The concentration of each sample solution was $50 \mu \mathrm{g} \mathrm{cm}^{-3}$. A gastight syringe (Hamilton Co., 81617) filled with these solutions was settled on the syringe pump (Harvard Apparatus, 11plus) and directly connected to the corona CAD. The flow rate of the mobile phase was set to $0.5 \mathrm{~cm}^{3} \mathrm{~min}^{-1}$. Average signal intensity from corona CAD during a three-minute experiment was used to analyze the 


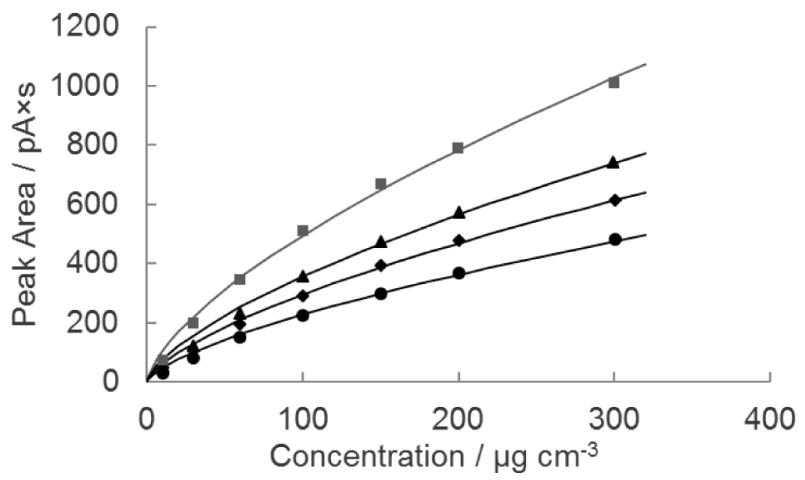

Fig. 1 Relationships between peak areas detected by corona CAD

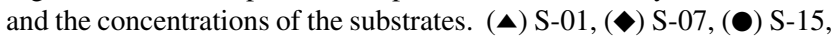
( $\square$ ) P-01. Solid lines show the fitted data to $2 / 3$ power of the concentrations of each substrate. The mobile phase was chloroform and the injected volume was $10 \mathrm{~mm}^{3}$.

effects of the densities. Toluene was measured every time between the sample analyses, and their average intensities were subtracted from those of the samples.

\section{Results and Discussion}

\section{Calibration curves in HPLC-corona CAD}

Figure 1 shows the relationships (calibration curves) between peak areas detected by corona $\mathrm{CAD}$ and the concentrations of some sample substrates (S-01, 07, 15 and P-01). The calibration curves for all substrates do not show linear relationships. As was reported in a number of papers, ${ }^{14,19,21,25-27}$ the slopes of the calibration curves for the substrates gradually decreased with increasing concentration. The signal intensity of the corona CAD should depend on the number of the electric charge. It is well known that the total electric charge is proportional to the surface area of a particle formed in the drying process. The surface area $A_{\mathrm{p}}$ of the particle produced in the corona CAD is calculated from its diameter $d_{\mathrm{p}}$ as follows:

$$
A_{\mathrm{p}}=\pi d_{\mathrm{p}}^{2}
$$

and $d_{\mathrm{p}}$ is given by the equation ${ }^{6}$

$$
d_{\mathrm{p}}=d_{\mathrm{d}}\left(\frac{c}{\rho_{\mathrm{p}}}\right)^{1 / 3}
$$

where $d_{\mathrm{d}}$ is the diameter of the original droplet (aerosol), $c$ the concentration of the eluted solution, and $\rho_{\mathrm{p}}$ the density of the substrate. $A_{\mathrm{p}}$ is thus calculated as follows:

$$
A_{\mathrm{p}}=\pi d_{\mathrm{p}}^{2}=\pi \cdot d_{\mathrm{d}}^{2} \cdot c^{23} \cdot \rho_{\mathrm{p}}^{-2 / 3}
$$

It is expected that the signal intensity of corona CAD is proportional to $A_{\mathrm{p}}$. Therefore, it should be proportional to $2 / 3$ power low of $c$ as was expected from Eq. (3). In Fig. 1, the black and gray curves were calculated by the least squares method with a $2 / 3$ power low of the relations between peak area and concentration for S-01, S-07, S-15 and P-01. The fact that the fitted curves and the observed peak intensities were in good agreement confirms the response of corona CAD is proportional to $2 / 3$ power low of the concentration of a substrate.

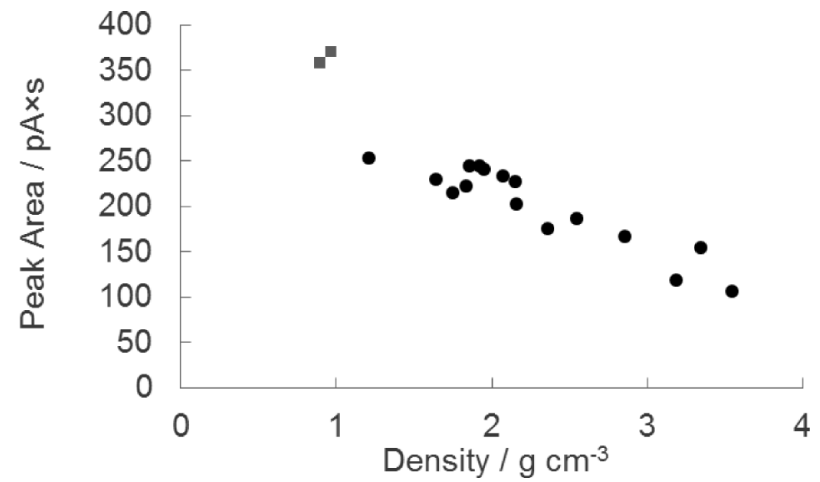

Fig. 2 Relationships between the peak areas in HPLC-corona CAD analysis and the densities of the injected substrates. The mobile phase was chloroform, injected volume was $10 \mathrm{~mm}^{3}$ and the concentrations

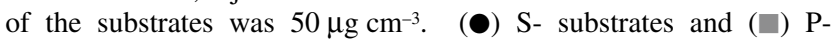
substrates.

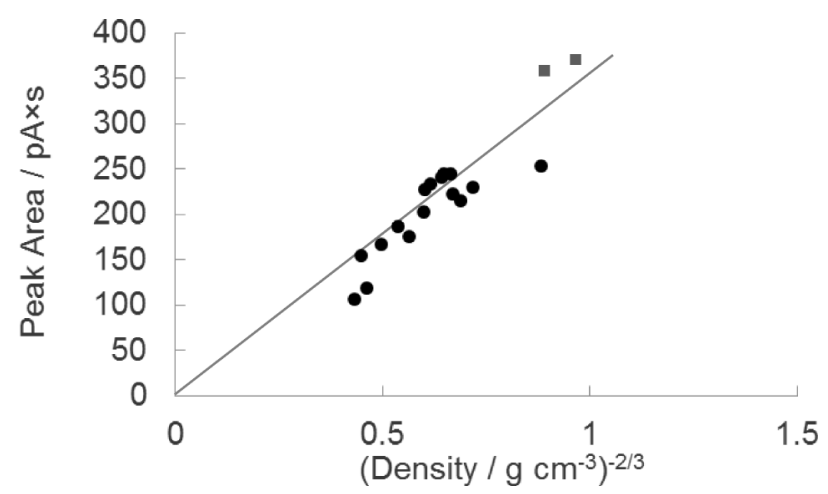

Fig. 3 Replotted data of Fig. 2 to show the dependencies of the peak areas to $-2 / 3$ power of the densities. (O) S- substrates and ( $\square$ ) Psubstrates.

\section{Relation between the signal intensity of corona $C A D$ and densities of substrates}

It should be also noted that the calibration curves for S-01, S-07, S-15 and P-01 in Fig. 1 were not universal. This result is inconsistent with previous literatures ${ }^{19,28}$ in which the corona CAD showed a nearly universal response independent of the substrates. In Fig. 1, the lighter density substrate showed higher response. This result corresponds well to Eq. (3) that suggests $A_{\mathrm{p}}$ (proportional to the intensity of corona CAD) is a decreasing function of density $\left(\rho_{\mathrm{p}}\right)$. Figure 2 shows the relationship between the peak areas in HPLC-corona CAD analysis and the densities for all of S- and P-substrates. This also clearly shows the observed peak areas of the substrates decrease with increasing density of the substrate.

If the surface charge density for these substrates were comparable, the peak areas could be correlated to the $(-2 / 3)$ power of the densities of the substrates based on the relation between the density of the substrate $\left(\rho_{\mathrm{p}}\right)$ and the surface areas $\left(A_{\mathrm{p}}\right)$ in Eq. (3). As shown in Fig. 3, the observed peak areas were almost proportional to the $(-2 / 3)$ power of the densities. Figure 4 shows similar relationships observed under different conditions for a) the concentration $\left.\left(150 \mu \mathrm{g} \mathrm{cm}^{-3}\right), \mathrm{b}\right)$ the injected volume $\left(1000 \mathrm{~mm}^{3}\right)$, and c) the solvent (toluene) with the injected volume of $1000 \mathrm{~mm}^{3}$. For the toluene solutions (Fig. 4c), the analyzed substrates were limited to S-03, 04 and 10 - 16. Regardless of the change in the conditions for Fig. 4, 

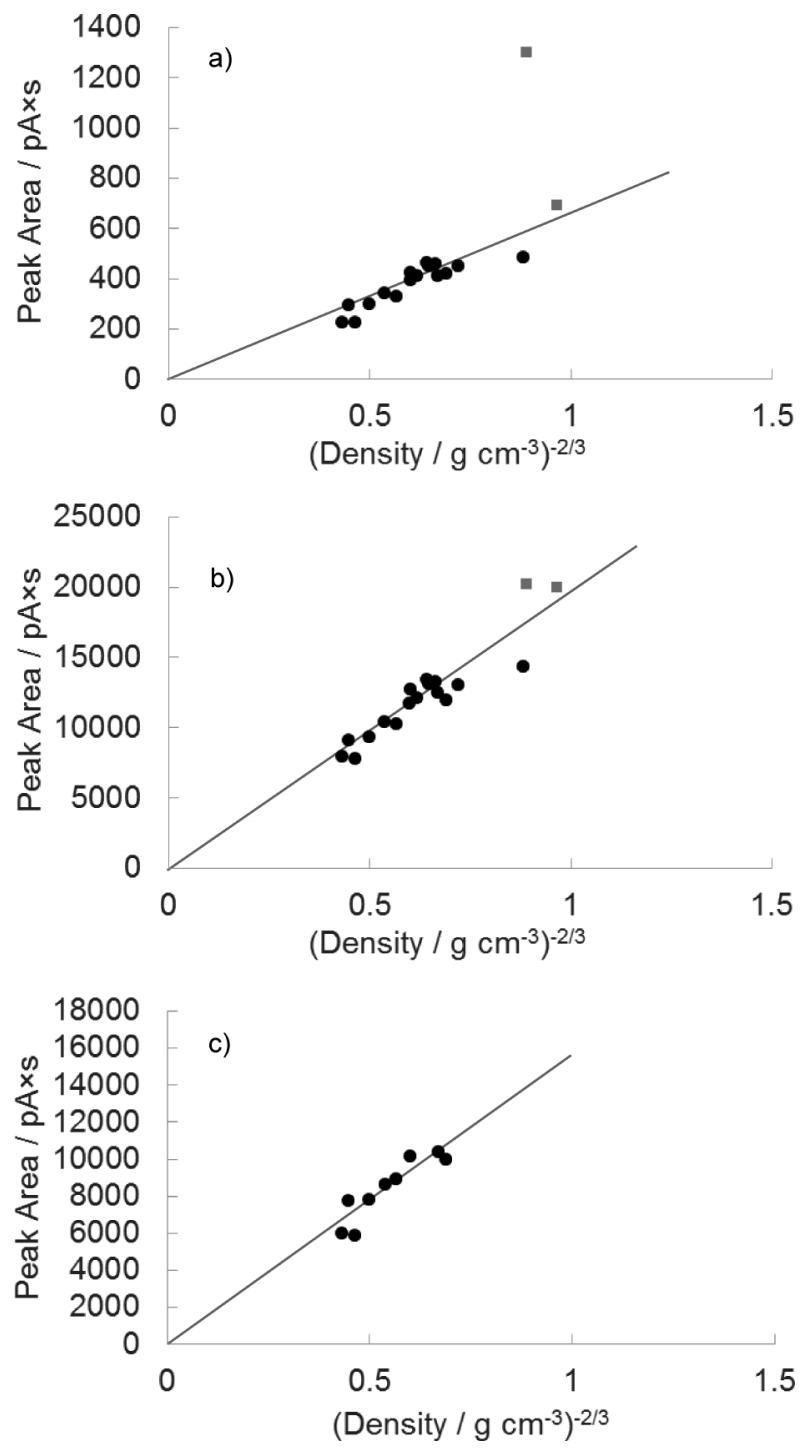

Fig. 4 Relationships between the peak areas monitored in HPLC-corona CAD and the densities of the injected substrates for various conditions. Injected volume, concentrations and the solvents were a) $10 \mathrm{~mm}^{3}, 150 \mu \mathrm{g} \mathrm{cm}^{-3}$, chloroform, b) $1000 \mathrm{~mm}^{3}, 50 \mu \mathrm{g} \mathrm{cm}^{-3}$, chloroform and c) $1000 \mathrm{~mm}^{3}, 50 \mu \mathrm{g} \mathrm{cm}^{-3}$, toluene, restrictively. S- Substrates, ( $\square)$ P- substrates.

the corona CAD responses were nearly proportional to the $(-2 / 3)$ power of the densities of the substrates. These results demonstrate that the response of corona CAD is mainly controlled with the density of the substrate.

\section{Intensity monitored by corona CAD using a syringe pump}

In the HPLC-corona CAD analysis, the concentration of the substrates in an eluted solution from HPLC is not generally constant in the corresponding chromatographic peak. In corona $\mathrm{CAD}$, solutions with different concentrations might generate different size particles and the response of the corona CAD could also change. ${ }^{6}$ Furthermore, even if the volume and the concentrations of injected solutions were equivalent, the elution profiles could be altered depending on the nature of the substrates. As a result, the eluted concentration at a time would be different from one substrate to another, which in turn might affect the corona CAD response. To compensate the effect of the variation in the elution profiles of the substrates, a constant

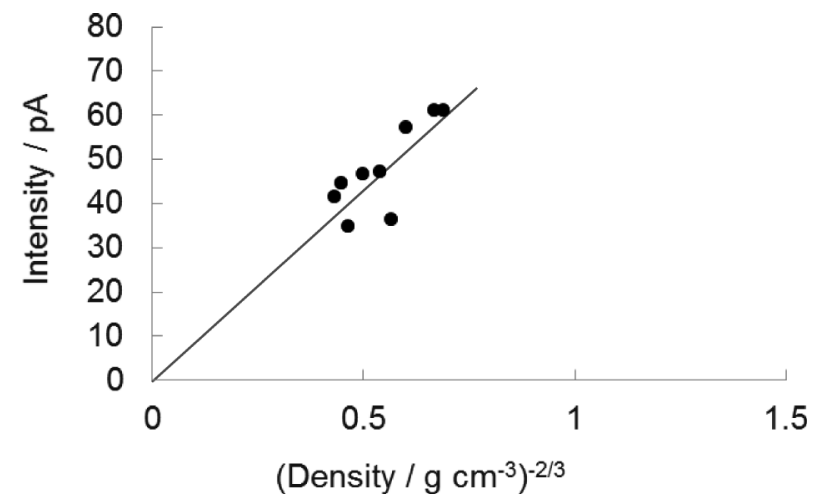

Fig. 5 Dependence of the average intensities of the corona CAD during the steady concentration flows to the densities of the S-03, 04 and $10-16$ substrates. The concentrations were $50 \mu \mathrm{g} \mathrm{cm}^{-3}$ and the solvent was toluene.

concentration flow of the substrate was introduced into the corona CAD by using a syringe pump. Figure 5 shows the relationship between the observed signal intensities of S-03, 04 and $10-16$ substrates monitored by corona CAD and the $(-2 / 3)$ power of the densities of the substrates using a syringe pump introduction. The observed intensities were also almost proportional to the $(-2 / 3)$ power of the density of the substrates injected constantly. These results further confirm that the signal intensity monitored by corona CAD is essentially dependent on the density of the substrate.

\section{Conclusions}

By using some brominated flame retardant and related substrates, it was suggested that the monitored intensities of the substrates by corona CAD were proportional to the $2 / 3$ power of the concentrations in the solutions and to the $(-2 / 3)$ power of the densities of the substrates. These results demonstrated that the response of corona $\mathrm{CAD}$ should be governed by the surface area of the substrate particles made through the drying process. It was proved that the universal response at a given, constant mobile phase for nonvolatile analytes reported by Górecki et al. ${ }^{19}$ might be applicable only for the substrates with a limited range of the densities.

\section{Acknowledgements}

We are deeply indebted to Dr. Jun-ichi Sugiyama (National Institute of Advanced Industrial Science and Technology) for helping with the analysis of the densities of frame retardants.

\section{References}

1. S. Almeling, D. Ilko, and U. Holtzgrabe, J. Pharm. Biomed. Anal., 2012, 69, 50.

2. C. F. Poole, "The Essence of Chromatography", 1st ed., 2003, Elsevier Science, Amsterdam.

3. J. M. Charlesworth, Anal. Chem., 1978, 50, 1414.

4. N. C. Megoulas and M. A. Koupparis, Crit. Rev. Anal. Chem., 2005, 35, 301

5. L. B. Allen and J. A. Koropchak, Anal. Chem., 1993, 65, 841. 
6. R. W. Dixson and D. S. Peterson, Anal. Chem., 2002, 74, 2930.

17. J. Shaodong, W. J. Lee, J. W. Ee, J. H. Park, S. W. Kwon, and J. Lee, J. Pharm. Biomed. Anal., 2010, 51, 973.

7. M. Senda, K. Fukushima, K. Hashiguchi, T. Matsumoto, P. H. Gamache, J. C. Waraska, and D. Asa, Chromatography, 2006, 27, 119.

8. K. Fukushima, K. Hashiguchi, T. Suzuki, M. Okawara, and Y. Sekiguchi, Chromatography, 2011, 32, 161.

9. M. M. Khandagale, J. P. Hutchinson, G. W. Dicinoski, and P. R. Haddad, J. Chromatogr. A, 2013, 1308, 96.

18. C. Merle, C. Laugel, P. Chaminade, and A. Baillet-Giffroy, J. Liq. Chromatogr. Relat. Technol., 2010, 33, 629.

19. T. Górecki, F. Lynen, R. Szucs, and P. Sandra, Anal. Chem., 2006, 78, 3186.

20. S. Matsuyama, S. Kinugasa, and H. Ohtani, Bunseki Kagaku, 2014, 63, 817.

21. D. Asa, Am. Lab., 2006, 38, 16.

10. J. P. Hutchinson, J. Li, W. Farrell, E. Groeber, R. Szucs, G. Dicinoski, and P. R. Haddad, J. Chromatogr. A, 2010, 1217, 7418.

11. K. Takahashi, S. Kinugasa, M. Senda, K. Kimizuka, K. Fukushima, T. Matsumoto, Y. Shibata, and J. Christensen, J. Chromatogr. A, 2008, 1193, 151.

12. D. Kow, G. Manius, S. Zhan, and H. P. Chokshi, J. Chromatogr. A, 2009, 1216, 5424.

13. U. Holtzgrabe, C. J. Nap, T. Beyer, and S. Almeiling, J. Sep. Sci., 2010, 33, 2402.

14. J. P. Hutchinson, J. Li, W. Farrell, E. Groeber, R. Szucs, G. Dicinoski, and P. R. Haddad, J. Chromatogr. A, 2011, 1218, 1646.

15. M. Psitorino and B. A. Pfeifer, Anal. Bioanal. Chem., 2008, 390, 1189.

16. I. Márquez-Sillero, S. Cádenas, and M. Valcárcel, Microchem. J., 2013, 110, 629.

22. Database of Reaxys, Compillation prepared by Reed Elsevier Properties https://www.reaxys.com/.

23. D. Schrader, "Polymer Handbook", ed. J. Brandrup, E. H. Immergut, and E. A. Grulke, 4th ed., 1999, John Wiley \& Sons, New York, V/91.

24. W. Wunderlich, "Polymer Handbook", ed. J. Brandrup, E. H. Immergut, and E. A. Grulke, 4th ed., 1999, John Wiley \& Sons, New York, V/87.

25. R. A. Moreau, Lipids, 2006, 41, 727.

26. J. P. Hutchinson, T. Remenyi, P. Neterenko, W. Farrell, E. Groeber, R. Szucs, G. Dicinoski, and P. R. Haddad, Anal. Chim. Acta, 2012, 750, 199.

27. C. Brunelli, T. Górecki, Y. Zhao, and P. Sandra, Anal. Chem., 2007, 79, 2472.

28. P. Sun, X. Wang, L. Alquier, and C. A. Maryanoff, $J$. Chromatogr. A, 2008, 1177, 87. 